\title{
Mesures de débit dans un écoulement à filets non parallèles
}

\section{Discharge measurements in non-parallel streamline flows}

\author{
PaR Marc SALVO
}

INGÉNIEUR AU DÉPARTEMENT ESSAIS DE LA DIREGTION DES ÉTUDES ET RECHERGHES D'ÉLECTRIGITÉ DE FRANCE

\begin{abstract}
La mesure des débits dans un écoulement à filets non parallèles se fait toujours avec des capteurs qui ne sont pas autocomposants; il s'ensuit que l'incertitude sur la direction de la vitesse induit une erreur sur la mesure du débit.

Il est montré que la connaissance de la réponse du capteur en fonction de l'angle capteur-filet liquide permet de minimiser cette erreur. Une application de cette theorie est faite pour des capteurs dont la réponse est $\cos ^{n} \varphi$ (moulinets hydrométriques). Dans ce cas, on peut trouver la forme de section de jaugeage la plus favorable.
\end{abstract}

\begin{abstract}
The use of non self-compounding gauging instruments for discharge measurements in nonparallel streamline flows results in measure ment errors, as the directions of the velocities are uncertain:

It is shown that this error can be reduced if the instrument response variations at different instrument/stream line angles are known.

The theory is then applied to instruments with a response of $\cos ^{n} \varphi$ (current meters). It is possible, in this case, to determine the optimum gauging section.
\end{abstract}

\section{POSITION DU PROBLÈME}

Dans un certain nombre de cas, on est amené à mesurer des débits dans un écoulement convergent, plus rarement divergent. Or, les capteurs de vitesse utilisés jusqu'ici ne sont généralement pas autocomposants, c'est-à-dire qu'ils n'indiquent pas la vitesse projetée sur la normale à la section de jaugeage, ou vitesse de débit, mais une grandeur fonction de l'angle que fait le filet fluide avec une direction privilégiée de l'appareil. Cet angle n'est jamais connu avec préci- sion; plus exactement, la direction des filets fluides ne peut être connue qu'approximativement, au moyen d'essais sur modèles, par exemple. Il découle de cette incertitude sur la position du vecteur vitesse, une erreur sur la mesure du débit.

Nous donnons une méthode générale pour minimiser cette erreur et nous l'étudions plus particulièrement dans le cas où le capteur est un moulinet. 


\section{A. - MÉthode GÉNÉRALE}

Soit un système d'axes Oxyz d'origine le point de mesure; le plan $x \mathrm{O} z$ est le plan tangent en $\mathrm{O}$ à la section de jaugeage; l'axe $O y$ est la normale en $O$ à cette section, orientée dans le sens général de l'écoulement. Soit D la droite passant par O supportant le vecteur vitesse. Soit enfin Ouvw un second trièdre trirectangle attaché au capteur, l'axe Ou supportant la direction privilégiée de ce dernier qu'on pourra admettre souvent comme axe de symétrie de révolution, $\mathrm{O} v$ et $\mathrm{O} w$ étant quelconques.

La réponse du capteur peut s'écrire, $m$ étant la mesure obtenue et $v$ la mesure de la vitesse:

$$
m=\mathrm{V} f(\alpha, \beta, \gamma)
$$

où $\alpha, \beta, \gamma$ sont les cosinus directeurs de $\mathrm{D}$ dans le système Ouviv. Considérons la surface $\mathrm{S}$, fixe dans le système Ouvw, d'équation polaire :

$$
p=\frac{V}{m}=\frac{1}{f(\alpha, \beta, \gamma)}
$$

Cette surface définit la: réponse du capteur; nous l'appellerons surface caractéristique.

Soit $\vec{v}$ le vecteur porté par $\mathrm{D}$ et de grandeur $p$; lorsque le système Ouvw, et donc le capteur, se déplace par rapport à la section de jaugeage, l'extrémité $\mathbf{P}$ de ce vecteur décrit dans les axes Ouvw la surface $\mathrm{S}$; le point $\mathrm{P}$ est le point caractéristique de la mesure.

La vitesse de débit $U$, mesure de la projection de $\vec{V}$ sur la normale $O y$ à la section de jaugeage est donnée par :

$$
\frac{\mathrm{U}}{m}=\vec{v} \cdot \vec{j}
$$

si $\vec{j}$ est le vecteur unitaire de $\mathrm{O} y$.

L'erreur relative sur $U$ est ainsi :

$$
\left|\frac{\Delta \mathrm{U}}{\mathrm{U}}\right|=\left|\frac{\overrightarrow{\Delta v} \cdot \vec{j}}{\vec{v} \cdot \vec{j}}\right|+\left|\frac{\Delta m}{m}\right|
$$

$\Delta m / m$ est l'erreur de lecture; elle est indépendante de la position du capteur. Il reste donc à minimiser la quantité

$$
\varepsilon=\left|\frac{\overrightarrow{\Delta v} \cdot \vec{j}}{\vec{v} \cdot \vec{j}}\right|
$$

$\varepsilon$ est nul, et donc minimal lorsque $\Delta \overrightarrow{v \cdot \vec{j}}=0$, c'est-à-dire lorsque $\dot{v}$ est orthogonal à $\mathrm{O} y$.
En fait, $\Delta \vec{v}$ est la somme de trois termes :

$\Delta \overrightarrow{v_{\omega}}$ : incertitude sur la position du capteur;

$\Delta \overrightarrow{v_{\mathrm{S}}}$ : incertitude sur la forme de la surface $\mathrm{S}$ (erreur d'étalonnage);

$\Delta \overrightarrow{v_{\mathrm{D}}}$ : incertitude sur la position de la vitesse.

Nous admettrons que les deux premiers termes sont indépendants de la position du capteur.

Il reste donc la condition suivante pour minimiser $\varepsilon$ :

$$
\Delta \overrightarrow{v_{\mathrm{D}}} \cdot \vec{j}=0
$$

Or, $\Delta \overrightarrow{v_{\mathrm{D}}}$ est contenu dans le plan tangent à $\mathrm{S}$ au point $\mathrm{P}$.

L'erreur sur la vitesse de débit est donc minimale lorsque la position du capteur est telle que le plan tangent au point représentatif de la mesure à la surface caractéristique du capteur soit parallèle au plan tangent à la section de jaugeage au point de mesure.

Cette conclusion est valable avec, comme seules hypothèses :

$1^{\circ}$ La surface caractéristique est indépendante de la grandeur de la vitesse, elle ne dépend que de sa direction par rapport au capteur;

$2^{\circ}$ L'erreur de lecture, l'erreur sur la position du capteur et l'erreur d'étalonnage sont indépendantes de Ia posititon du point $P$ sur la surface caractéristique.

\section{Remarque :}

Le point $\mathrm{P}$ sur la surface $\mathrm{S}$, et done la position du capteur (axes Ouvw), ne sont pas uniques pour une même position de la vitesse (D) par rapport aux axes O'xyz. En effet, soit $\psi$ l'angle $\left(\mathrm{O}_{y}, \mathrm{D}\right)$; cherchons à déterminer la position des axes $\mathrm{O} x y z$. Un point $M$ de $S$ est un point $P$ à la seule condition suivante : la droite OM fait l'angle $\psi$ avec la normale en $P$ à la surface $S$; on peut alors tracer $\mathrm{O} y$ qui est la parallèle menée par $\mathrm{O}$ à cette normale; les axes $\mathrm{O} x, \mathrm{O} z$ sont alors déterminés, puisque la droite $\mathrm{D}$ est connue (c'est $\mathrm{OM}$ ).

Les points $\mathrm{P}$ forment donc le lieu des points de $\mathrm{S}$ où la normale fait un angle constant avec une direction fixe $(\mathrm{O} g)$.

En particulier, si $S$ est de révolution autour de $\mathrm{O} u$, les points $\mathrm{P}$ décrivent des parallèles d'axe Ou. 


\section{B. - APPLICATION AUX MOULINETS HYDROMETTRIQUES}

Nous allons refaire le calcul d'erreur en précisant la forme de la fonction caractéristique.

\section{Généralités.}

Soit $\mathrm{O}$ le point de mesure; $x \mathrm{O} z$ est le plan tangent en $\mathrm{O}$ à la section de jaugeage; $\mathrm{O} y$ est la normale à cette section.

Oru est l'axe du moulinet; Ow est confondu avec $O x$, que nous supposerons de plus horizontal. Ces choix correspondent au fait que les moulinets sont ordinairement montés sur une perche horizontale ( $\mathrm{O} x, \mathrm{O} w$ ), et qu'il n'est possible que de leur fournir un mouvement de rotation autour de cette perche.

Les angles sont définis par la figure 1, et l'on peut écrire les deux relations :

$$
\begin{aligned}
& \cos \alpha=\cos \tau \cos \theta, \\
& \cos \varphi=\cos \tau \cos (\theta-\omega) .
\end{aligned}
$$

La vitesse de débit est donc:

$$
\mathrm{U}=\mathrm{V} \cos \tau \cos \theta
$$

Soit $f(\varphi)$ la fonction caractéristique du moulinet; nous supposons que cette fonction ne dépend pas de la grandeur de la vitesse, et que le moulinet admet la symétrie de révolution autour de son axe. La mesure est donc :

$$
m=\mathrm{V} f(\varphi)
$$

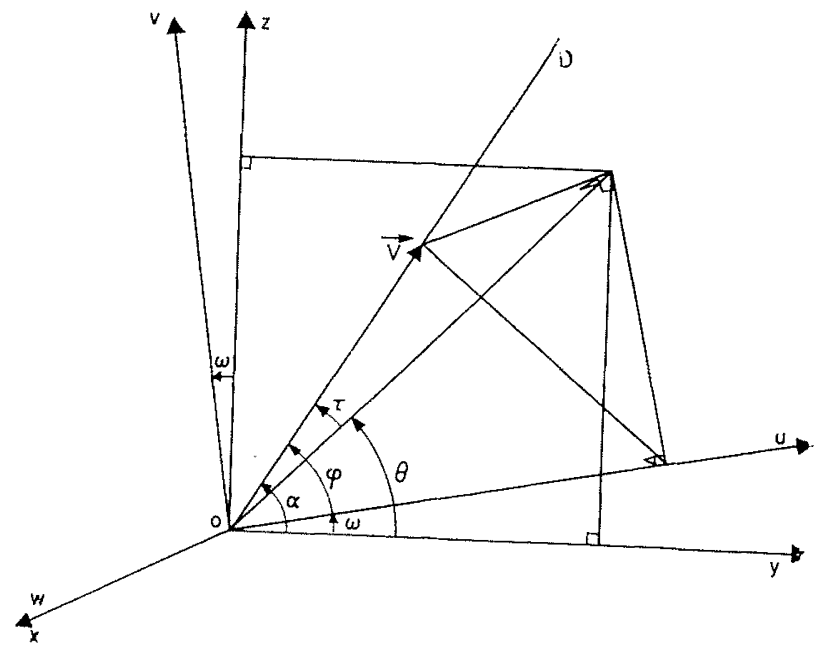

FIG. 1
La vitesse de débit est donc

$$
\mathrm{U}=m \frac{\cos \tau \cos 0}{f(\varphi)}
$$

D'où

$$
\frac{\Delta \mathrm{U}}{\mathrm{U}}=\frac{\Delta m}{m}-\operatorname{tg} \theta \Delta \theta-\operatorname{tg} \tau \Delta \tau-\frac{\Delta f}{f}
$$

\section{Hypothèses et poursuite du calcul.}

Pour aller plus loin, il faut expliciter le terme $\Delta f / f$; il est donc nécessaire de faire une hypothèse sur la forme de la fonction $f$. Nous supposerons que $f(\varphi)=\cos ^{n} \varphi$.

Alor's :

$$
\frac{\Delta f}{f}=n \frac{\Delta \cos \varphi}{\cos \varphi}+\varepsilon_{f}
$$

où $\varepsilon_{f}$ est la précision de l'étalonnage.

On aboutit ainsi à :

$$
\begin{aligned}
\left|\frac{\Delta U}{U}\right| & =|-\operatorname{tg} \theta+n \operatorname{tg}(\theta-\omega)| \Delta \theta \\
& +|(n-1) \operatorname{tg} \tau| \Delta \tau+n \operatorname{tg}(\theta-\omega) \mid \Delta \omega \\
& +\varepsilon_{i}+\left|\frac{\Delta m}{m}\right|
\end{aligned}
$$

Résumons ici les hypothèses pour lesquelles cette formule est valable :

a) $\Delta m / m$ est indépendant de $\varphi$;

b) $\varepsilon_{f}$ est indépendant de $\varphi$;

c) La caractéristique du moulinet est de révolution, et est de la forme $f(\varphi)=\cos ^{n} \varphi$, indépendante de la grandeur de la vitesse.

Ceci étant, nous pouvons faire les remarques suivantes :

$1^{\circ} \Delta m / m$ et $\varepsilon_{f}$ ne sont pas à prendre en compte pour minimiser $\Delta U / U$ en vertu des hypothèses $a$ et $b$.

$2^{\circ}$ Nous admettrons que $\Delta(\omega)=0$.

$3^{\circ}$ Le seul degré de liberté des moulinets étant une rotation autour de $\mathrm{O} x$, on n'a aucune action sur le coefficient de $\Delta \tau$.

Ainsi l'erreur sera minimale en même temps que la quantité :

$$
\varepsilon=|-\operatorname{tg} \theta+n \operatorname{tg}(\theta-\omega)|
$$




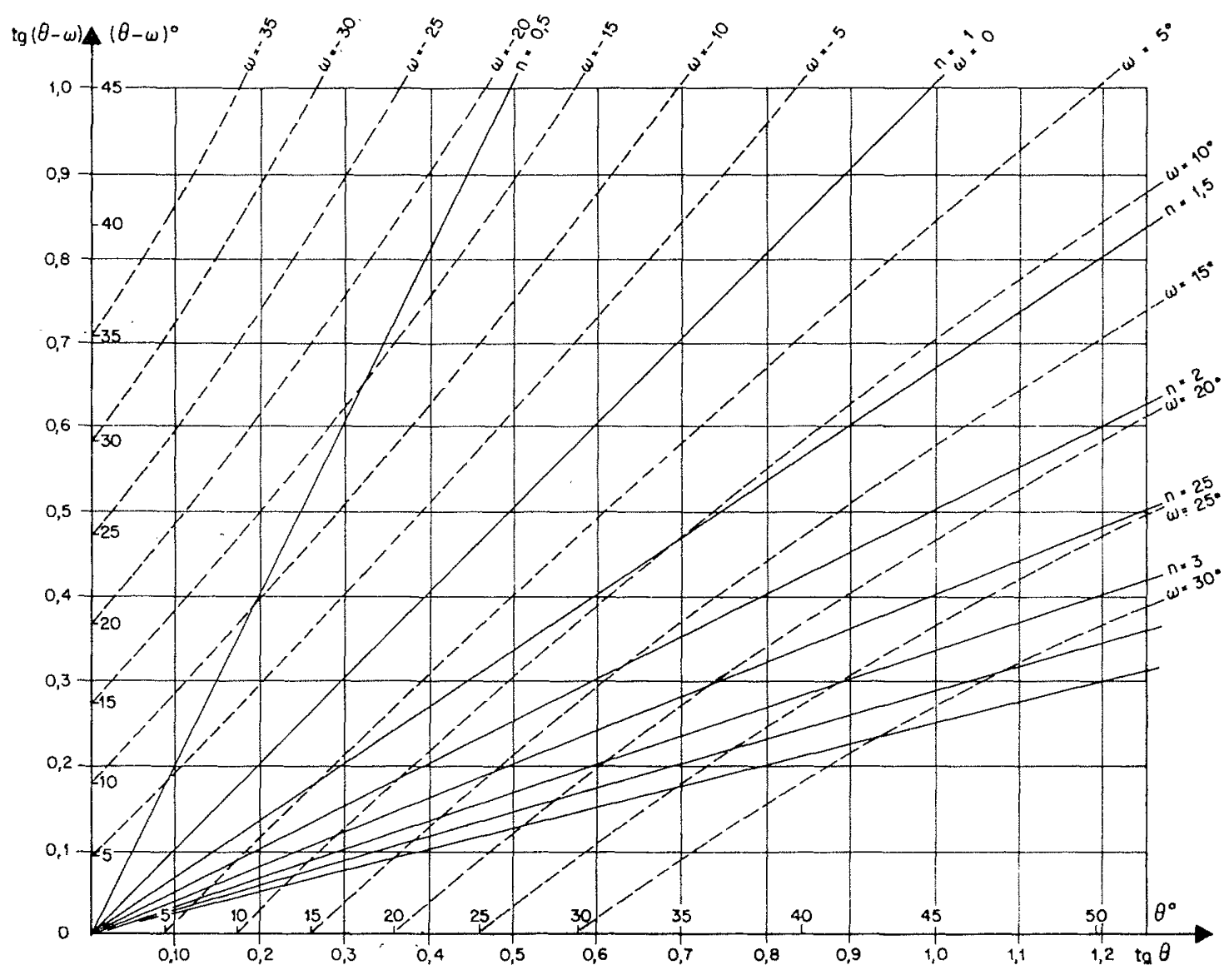

Fig. 2

Abaque pour la détermination de $\omega$.

\section{Résolution par abaque.}

On peut représenter $\varepsilon$ à l'aide de l'abaque figure 2 . On a porté en ordonnées tg $(\theta-\omega)$ et en abscisse $\operatorname{tg} \theta$, suivant une échelle linéaire, les angles $(\theta-\omega)$ et $\theta$ étant figurés en regard des valeurs de leurs tangentes.

Dans ces axes, le point $M$ caractérisant les conditions de la mesure se trouve à l'intersection des droites

$$
(\theta-\omega)=\mathrm{Cte} \quad \text { et } \theta=\mathrm{Cte} ;
$$

par ce point passe la branche d'hyperbole définie par $\omega=$ Cte; enfin les droites issues de l'origine ont pour équation :

$$
-\operatorname{tg} \theta+n \operatorname{tg}(\theta-\omega)=0
$$

C'est-à-dire $\varepsilon=0$; leur pente est $\mathrm{I} / n$.

Pour le point $M$, la valeur de $\varepsilon / \Delta \theta$ est mesurée par la longueur du segment MM', parallèle à $^{\prime}$ l'axe des abscisses et compris entre les courbes $\omega=$ Cite et $n=$ Cte, correspondant à la position du moulinet $(\omega)$ et à sa caractéristique $(n)$.
Ainsi, $\varepsilon=0$, et $\Delta U / U$ est minimal, lorsque le point figuratif $\mathrm{M}$ se trouve sur la droite $n=$ Cte correspondant au moulinet utilisé.

Notons que cela détermine un angle $\omega$ et un seul, donc une seule position du moulinet, pour un angle $\theta$ donné. Dans le cas général, nous avions obtenu un ensemble de positions possibles; cela tient au fait que nous avons restreint la liberté de mouvement du capteur.

\section{Discussion.}

Les calculs précédents se rapportent à un capteur isolé. Lors d'un jaugeage, on utilise en général une batterie de moulinets montés sur une perche horizontale; les axes de ces moulinets sont tous dans un même plan. Il s'ensuit que les sections de jaugeage sont nécessairement cylindriques. L'angle $\omega$ est le même pour tous les moulinets, mais l'angle $\theta$ (et l'angle $\tau$ ) varie en général de l'un à l'autre.

On considèrera donc l'angle $\theta_{m}$ moyen par exemple, et l'on placera les moulinets à l'angle (1) tel que $\varepsilon=0$ pour cet angle $0_{m}$ fictif, l'écart maximal $0-\theta_{m}$ étant $\psi$. 


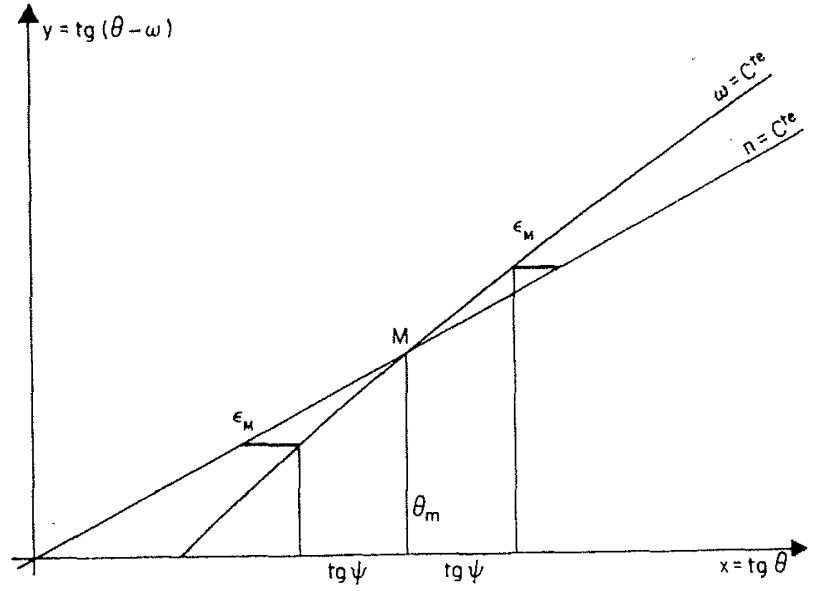

FIG. 3
Soit $y^{\prime}$ la pente de l'hyperbole $\omega=$ Cte; on peut écrire comme le montre la figure 3, que l'erreur maximale est donnée sensiblement par :

$$
\frac{y^{\prime} \operatorname{tg} \psi}{\varepsilon_{M}+\operatorname{tg} \psi}=\frac{1}{n}
$$

Soit :

$$
\varepsilon_{M}=\left(n y^{\prime}-1\right) \operatorname{tg} \psi
$$

L'équation de la courbe $\omega=$ Cte est :

$$
\begin{gathered}
y=\operatorname{tg}(\theta-\omega) \\
x=\operatorname{tg} \theta
\end{gathered}
$$

ou :

$$
y=\frac{x-\operatorname{tg} \omega}{x \operatorname{tg} \omega+1}
$$

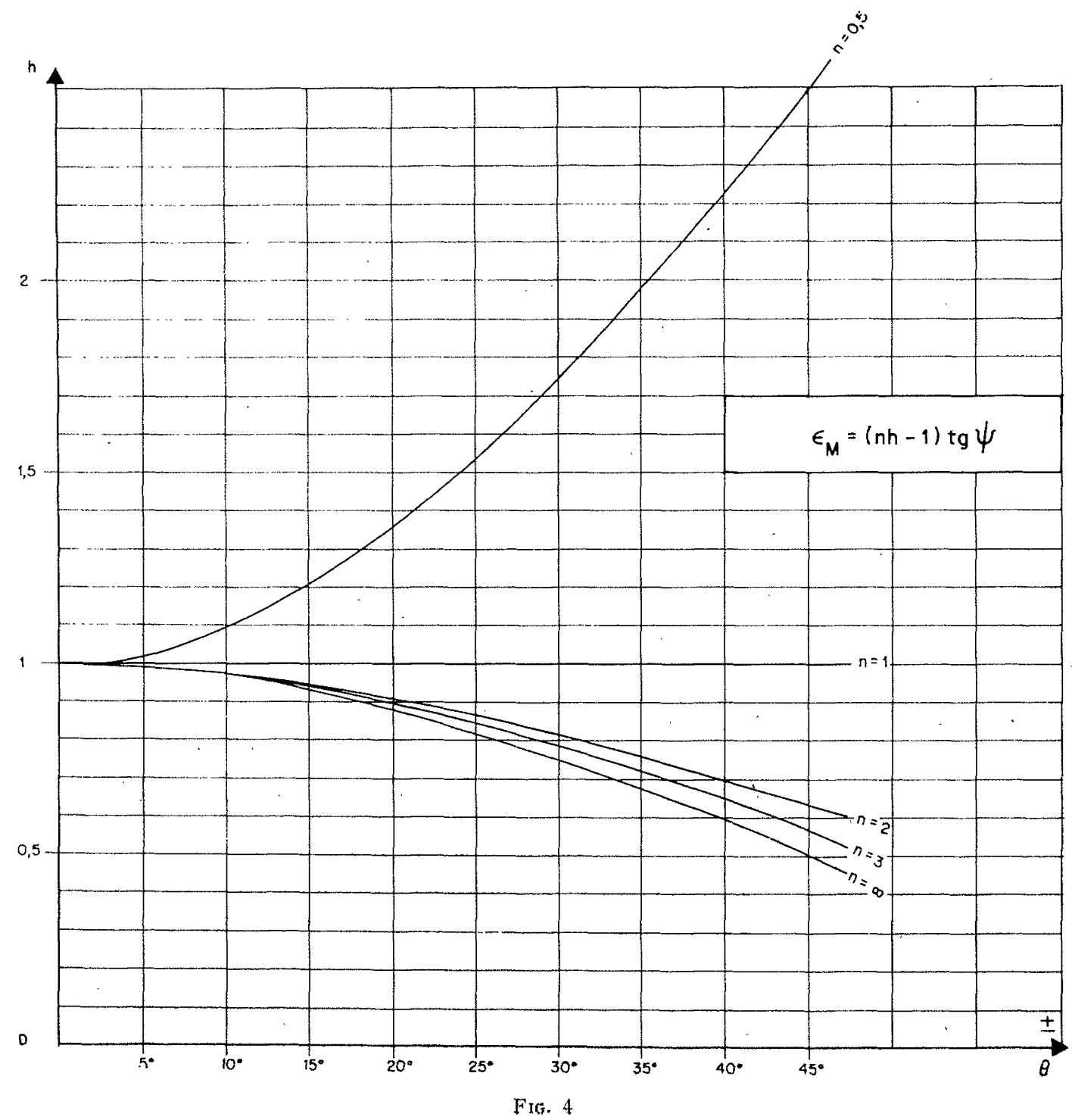


d'où l'on tire :

$$
y^{\prime}=\frac{1+\operatorname{tg}^{2} \omega}{(1+x \operatorname{tg} \omega)^{2}}
$$

De sorte que :

$$
\varepsilon_{M}=\left(n \frac{1+\operatorname{tg}^{2} \omega}{\left(1+\operatorname{tg} \theta_{m} \operatorname{tg} \omega\right)^{2}}-1\right) \operatorname{tg} \psi
$$

Cette formule ne préjuge en rien de la forme de la section de jaugeage.

Compte tenu du choix de $\omega$ par rapport à $\theta_{m}$, nous pouvons l'écrire :

$$
\varepsilon_{\mathrm{M}}=\left[n \frac{n^{2}+\operatorname{tg}^{2} \theta_{m}}{n^{2}+n^{2} \operatorname{tg}^{2} \theta_{m}}-1\right] \operatorname{tg} \psi
$$

Pour étudier $\varepsilon_{\mathrm{M}}$ en fonction de $n$, et $\theta_{m}$ (qui fixe la forme de la section de jaugeage) nous examinerons la quantité :

$$
h=\frac{n^{2}+\operatorname{tg}^{2} \theta}{n^{2}+n^{2} \operatorname{tg}^{2} \theta}
$$

Le graphique figure 4 représente les variations de $h$. On voit que, pour des valeurs de $n \geqslant 1$, on a intérêt à ce que $\theta$ soit grand. Or la formule (2) montre qu'il existe un cas simple où $\varepsilon$ est toujours nul; c'est lorsque $\theta=0$, $\omega=0$; c'est-à-dire que la section de jaugeage est normale à la direction générale de l'écoulement en chaque point, et les moulinets sont normaux à cette section (cette forme de section généralise les surfaces équipotentielles dans un écoulement potentiel); cette solution est séduisante, car elle est indépendante de $n$; par contre, la réalisation d'une telle section est compliquée, et le jaugeage est plus malaisé que si l'on a une section plane. De plus, les équations (3) et (4) et le graphique 4 montrent que cette solution est plus défavorable que celle de la section plane, puisque $h=1$ quel que soit $n \lessgtr 1$. La complication de cette solution, a priori élégante, ne serait rentable que dans le cas de moulinets pour lesquels $n$ est inférieur à 1 ; dans ce cas, en effet, $h$ croît très vite en fonction de $\theta$, et l'on aurait intérêt à se placer de telle sorte que $\theta=0$.

\section{Conclusions.}

Soit un écoulement à filets non parallèles; nous voulons mesurer le débit à l'aide de moulinets dont la caractéristique est de révolution, indépendante de la grandeur de la vitesse et de la forme $f(\varphi)=\cos ^{n} \varphi$. Nous faisons l'hypothèse supplémentaire que seul le terme correspondant à l'incertitude sur la position de la vitesse dépend de l'angle o. Nous pouvons alors choisir, grâce à l'abaque indiqué, la position des moulinets de manière que l'erreur due à cette incertitude soit minimale. De plus, nous pouvons dive que la section plane est plus favorable en général qu'une section qui serait orthogonale en chaque point à la direction moyenne de l'écoulement.

\section{DIRECTION HYDRAULIQUE REPUBLIQUE MALI}

recherche avant fin Mars :

\section{DEUX INGÉNIEURS}

confirmés, respectivement pour Etudes aménagements régionaux et Hydraulique urbaine suivant contrat ou convention avec Société Résidence à Bamako - Logement assuré 